\title{
Relationship between low blood pressure and renal/cardiovascular outcomes in Japanese patients with chronic kidney disease under nephrologist care: the Gonryo study
}

\author{
Tae Yamamoto - Masaaki Nakayama • Mariko Miyazaki • \\ Masato Matsushima - Toshinobu Sato • Yoshio Taguma • \\ Hiroshi Sato $\cdot$ Sadayoshi Ito
}

Received: 8 August 2014/ Accepted: 13 January 2015/Published online: 4 February 2015

(C) Japanese Society of Nephrology 2015

\begin{abstract}
Background Previous studies established a J-shaped association between blood pressure (BP) and cardiovascular disease (CVD) in chronic kidney disease (CKD), and the different clinical profiles of CVD by ethnicity. However, the adequately lower BP target remains unclear in Asian patients with CKD.

Methods This prospective observational study included 2,655 Japanese outpatients with CKD under nephrologist care who met the inclusion criteria, namely estimated glomerular filtration rate $<60 \mathrm{~mL} / \mathrm{min}$ and/or presenting proteinuria. The patients were divided by $10-\mathrm{mmHg}$ BP
\end{abstract}

T. Yamamoto $(\square) \cdot$ M. Miyazaki $\cdot$ S. Ito

Division of Nephrology, Endocrinology and Vascular Medicine,

Tohoku University Graduate School of Medicine, 1-1

Seiryomachi, Aoba-ku, Sendai, Miyagi 980-8574, Japan

e-mail: tae.yamamoto@med.tohoku.ac.jp

M. Nakayama - M. Miyazaki - M. Matsushima · Y. Taguma ·

H. Sato $\cdot$ S. Ito

Center for Advanced Integrated Renal Science, Tohoku

University Graduate School of Medicine, Sendai, Japan

M. Nakayama

Division of Nephrology, Endocrinology Vascular Medicine and

Diabetology, Fukushima Medical University, Fukushima, Japan

M. Matsushima

Department of Clinical Research, The Jikei University School of

Medicine, Tokyo, Japan

T. Sato $\cdot$ Y. Taguma

Kidney Center, Japan Community Health Care Organization,

Sendai Hospital, Sendai, Japan

H. Sato

Clinical Pharmacology and Therapeutics, Tohoku University

Graduate School of Pharmaceutical Sciences, Sendai, Japan increments by clinical data. The end points were death, cardiovascular events (CVEs), and end-stage kidney disease (ESKD) that requires renal replacement therapy.

Results During a 3.02-year median follow-up, 64 patients died, 120 developed CVEs, and 225 progressed to ESKD. In the adjusted Cox models, the risks of CVEs and allcause mortality were higher in the patients with systolic BPs (SBPs) $<110 \mathrm{mmHg}$ than in those with SBPs of $130-139 \mathrm{mmHg}$. Moreover, the risk was higher in those with diastolic BPs (DBPs) $<70 \mathrm{mmHg}$ than in those with DBPs of $80-89 \mathrm{mmHg}$. Although $\mathrm{SBPs} \geq 140 \mathrm{mmHg}$ were associated with higher incidence rates of ESKD, no significant increased risk was associated with BPs $<130$ / $80 \mathrm{mmHg}$.

Conclusions $\quad$ SBPs $<110 \mathrm{mmHg}$ and DBPs $<70 \mathrm{mmHg}$ were independent risk factors of CVEs and all-cause mortality. No lower BPs were observed as significant risk factors of progression to ESKD. This study suggests that the lower BP target in Asian patients with CKD should be $\geq 110 / 70 \mathrm{mmHg}$.

Keywords Blood pressure - Cardiovascular disease . Hypertension · Kidney failure $\cdot$ Outcome $\cdot$ Proteinuria

\section{Introduction}

Chronic kidney disease (CKD) has become a worldwide public health problem because it increases the risk of progression to end-stage kidney disease (ESKD), excess cardiovascular morbidities, and premature death [1]. Hypertension is common in patients with CKD, and it is a strong risk factor of poor outcomes in these patients [2]. As for the optimal blood pressure (BP) in CKD patients, the recommended target systolic BP (SBP)/diastolic BP (DBP) 
is $<140 / 90 \mathrm{mmHg}$ for those without albuminuria and $<130 / 80 \mathrm{mmHg}$ for those with urine albumin excretion rates $>30 \mathrm{mg} / 24 \mathrm{~h}$, according to the Kidney Disease: Improving Global Outcomes (KDIGO) Clinical Practice Guidelines [3]. The recommendations were derived from previous data such as those from the MDRD (Modification of Diet in Renal Disease) study and the AASK (AfricanAmerican Study of Kidney Disease and Hypertension), which demonstrated that aggressive BP control to lower BP targets was associated with a lower risk of CKD progression, especially in patients with proteinuria [4, 5]. However, the lower limit of the optimal BP target range for patients with CKD remains unclear. This needs to be addressed because a J-shaped association between BP, and morbidity and mortality events has been shown in a broad range of populations, including CKD patients [6-8].

Therefore, 2 aspects of the issue regarding BP in CKD need to be clarified, namely the risk of renal and nonrenal events. A recent meta-analysis concerning CKD concluded that although an intensive BP-lowering strategy reduced the risk of renal outcomes, it had no clear effect on the risk of cardiovascular events (CVEs) and death [9], indicating a gap between the optimal BP in terms of renal and nonrenal outcomes (cardiac disease, stroke, and premature death) in CKD populations. This is particularly important in terms of ethnicity because of the differences in the type of cardiovascular disease (CVD) between Western and Asian populations [10], namely the high prevalence of cardiac disorders in the former and stroke in the latter. Moreover, the stronger association between BP and CVD in Asian populations than in Caucasian populations has been reported [11]. In fact, an $\mathrm{SBP}<120 \mathrm{mmHg}$ has been associated with a further reduced risk of CVD mortality in the Asian general population, unlike in Caucasian populations [12, 13]. Therefore, we examined the relationship between BP and incidence of CVEs, all-cause mortality, and progression to ESKD in a Japanese cohort with CKD.

\section{Methods}

\section{Participants}

All the participants were under regular nephrologist care. We conducted a prospective observational cohort study, named the Gonryo CKD Project, in an outpatient basis in 11 hospitals that offer nephrology services, including one university hospital in Miyagi Prefecture, located in the northeast area of Japan [14, 15]. The participating hospitals covered almost the entire medical network of the area. Between May 2006 and November 2008, outpatients under nephrologist care were invited to participate. Thus, 4,015 patients who provided informed consent were enrolled in the study [14]. The institutional review board of Tohoku University School of Medicine (No. 2006-10, UMIN000011211) and the respective participating hospitals approved the study protocol. Based on the previous publications of the study, certain subjects were excluded for the following reasons: lack of data on serum creatinine levels $(n=145)$, unknown underlying renal disease ( $n=170)$, no-show $(n=1)$, and estimated glomerular filtration rate $(\mathrm{eGFR})>60 \mathrm{~mL} / \mathrm{min}$ without proteinuria $(n=888)$ or no urinary testing results $(n=117)$. After exclusion of those without BP data $(n=39), 2,655$ patients who met the CKD criteria with BP data were evaluated in the present study (Fig. 1).

\section{Patient classification and primary outcomes}

The patients were classified according to baseline BP. SBP was divided into 6 categories by $10-\mathrm{mmHg}$ increments as follows: <110 (S1), 110-119 (S2), 120-129 (S3), 130-139 (S4), 140-149 (S5), and $\geq 150 \mathrm{mmHg}$ (S6 group). DBP was divided into 4 categories as follows: $<70$ (D1), 70-79 (D2), 80-89 (D3), and $\geq 90 \mathrm{mmHg}$ (D4). The reference ranges for SBP and DBP were $130-139 \mathrm{mmHg}$ (S4) and 80-89 mmHg (D3), respectively. Elderly patients were defined as those aged $\geq 65$ years, and diabetes patients included those treated for diabetes, not only diabetic nephropathy (DN). We classified the underlying renal diseases as previously described [14]. Briefly, primary renal diseases were defined by primary glomerulonephritis, tubule-interstitial nephritis, minimal change in nephrotic syndrome, and acute glomerulonephritis, including biopsy-

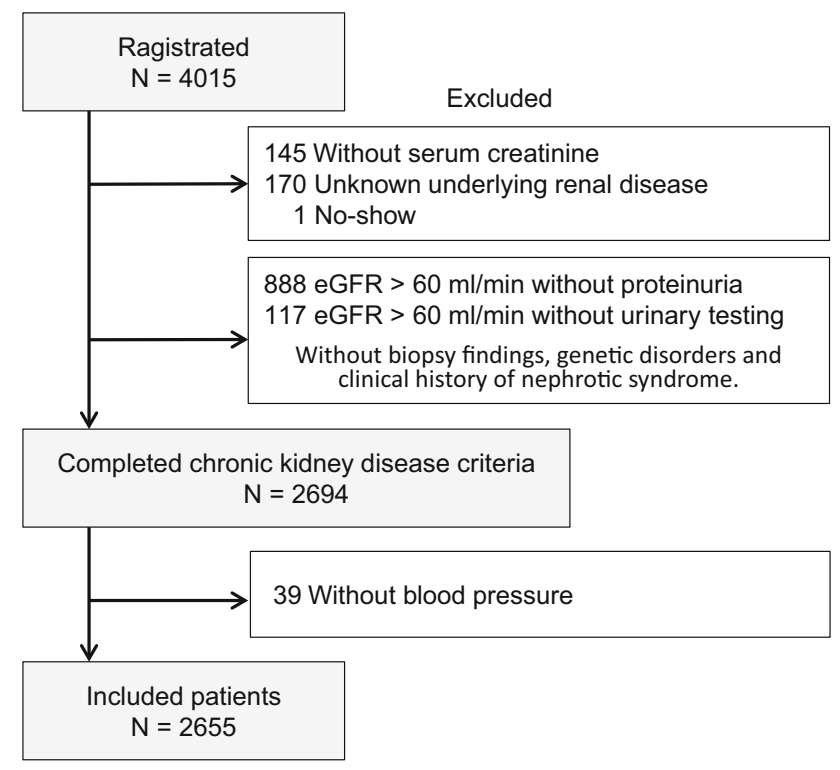

Fig. 1 A flowchart representing the patients' inclusion into the present study 
proven renal disease in $81 \%$ of the patients. Hypertensive nephropathy (HN) was defined by a history of hypertension in the absence of other possible disorders, including biopsy findings of nephrosclerosis in $21 \%$ of the patients. DN, including biopsy-proven disease in $25 \%$ of the patients, was defined by a history of diabetes accompanying nephropathy, including cases with biopsy findings of DN or patients who presented with nephropathy together with diabetic retinopathy. Other nephropathies $(\mathrm{ON})$, including biopsy-proven disease in $54 \%$ of the patients, were defined by ON not included in the other 3 groups as follows: systemic or secondary renal disorders such as systemic vasculitis, collagen diseases, infectious diseases (e.g., hepatitis $\mathrm{B}$ or $\mathrm{C}$ virus infection), drug use, pregnancy, vascular disorders, urological disorders, and genetic disorders, including polycystic kidney disease.

Primary outcomes were CVEs, all-cause death before renal replacement therapy, or ESKD requiring renal replacement therapy. CVEs included angina pectoris (AP), acute myocardial infarction (AMI), congestive heart failure (CHF), and stroke (cerebral bleeding and infarction). The outcomes after study enrollment were determined from the hospital medical records, death certificates, and interviews with the attending physicians at the time of the annual checkups, until March 10, 2011, or until the end of a 5-year follow-up (whichever came first). A CVE was defined as a disease of the circulatory system (International Classification of Disease, 10th Revision: I00-I99), and the patients with AP or AMI included those who had received coronary stenting, angioplasty, or bypass operation or had definite clinical course of AMI. Among the patients with CHF, only those who needed admission for treatment were counted. Diagnoses of stroke and stroke subtypes were based on the Classification of Cerebrovascular Diseases III published by the National Institute of Neurological Disorders and Stroke [16], and only patients whose conditions were confirmed by computed tomography or brain magnetic resonance imaging were included. Asymptomatic cerebral infarction or lacunar infarct was not included.

\section{Data collection}

Data on patient were obtained from the medical records at each hospital. Information on outcomes, laboratory findings, and all other follow-up data were collected annually until the end of follow-up, that is, death, initiation of renal replacement therapy, or 5 years after registration. BP data were obtained with the patient in the sitting position, using an automatic sphygmomanometer based on the Korotkoff sound technique. The BP data were collected at outpatient clinics attached to the local medical centers. We used the urine and blood examination results in each participating hospital as laboratory data. Serum creatinine levels were measured by using the enzyme assay method and monitored annually. We estimated the GFR by using the formula for the Japanese population $\left(\mathrm{mL} / \mathrm{min} / 1.73 \mathrm{~m}^{2}\right)$ [17]. The definition of urine protein extraction was a positive dipstick test for spot urine, which corresponded to values $>30 \mathrm{mg} / \mathrm{dL}$ by the urine analyzer in each hospital. Moreover, we identified an exposure to antihypertensive medications, which included different mechanisms of action, such as angiotensin-converting enzyme inhibitors or angiotensin-receptor blockers, calcium-channel blockers, $\alpha$-blockers, $\beta$-blockers, diuretics, and other agents such as vasodilators.

\section{Statistical analysis}

Data are shown as mean \pm standard deviation (SD) values, unless otherwise specified. A $p<0.05$ indicated statistical significance. Differences between groups were analyzed by using one-way analysis of variance (ANOVA), followed by a post hoc test if significant in the ANOVA analysis. Correlations were calculated by using the nonparametric Spearman rank test. In the survival analysis, the outcome for renal events was determined from the progression to ESKD and that for nonrenal events was determined from the CVEs and all-cause mortality (combined CVEs). The results of the Cox regression analysis were presented as hazard ratios (HRs) with $95 \%$ confidence intervals $(95 \%$ CIs). All data were statistically analyzed by using Stata version 12.1 (Stata Corp LP, College Station, Texas, USA).

\section{Results}

Baseline characteristics among BP categories

The mean SBP and DBP values in the 2,655 CKD patients were $131 \pm 16$ and $77 \pm 11 \mathrm{mmHg}$, respectively. Table 1 summarizes the features and selected baseline variables of each SBP or DBP category by $10-\mathrm{mmHg}$ increments. Patients with lower SBP were younger, had a lower prevalence of proteinuria and a higher eGFR. Patients with lower DBP were older, with a higher prevalence of proteinuria and DN. The eGFR did not differ between the DBP categories. SBP and DBP were closely associated ( $\rho=0.606, p<0.0001)$. SBP correlated positively with age $(\rho=0.216, p<0.0001)$ and pulse pressure (PP; $\rho=0.735, \quad p<0.0001)$, and negatively with eGFR ( $\rho=-0.174, p<0.0001)$, whereas DBP correlated negatively with age $(\rho=0.121, p<0.0001)$ and did not correlate with PP $(\rho=-0.03$, ns) or eGFR $(\rho=-0.02$, ns). 


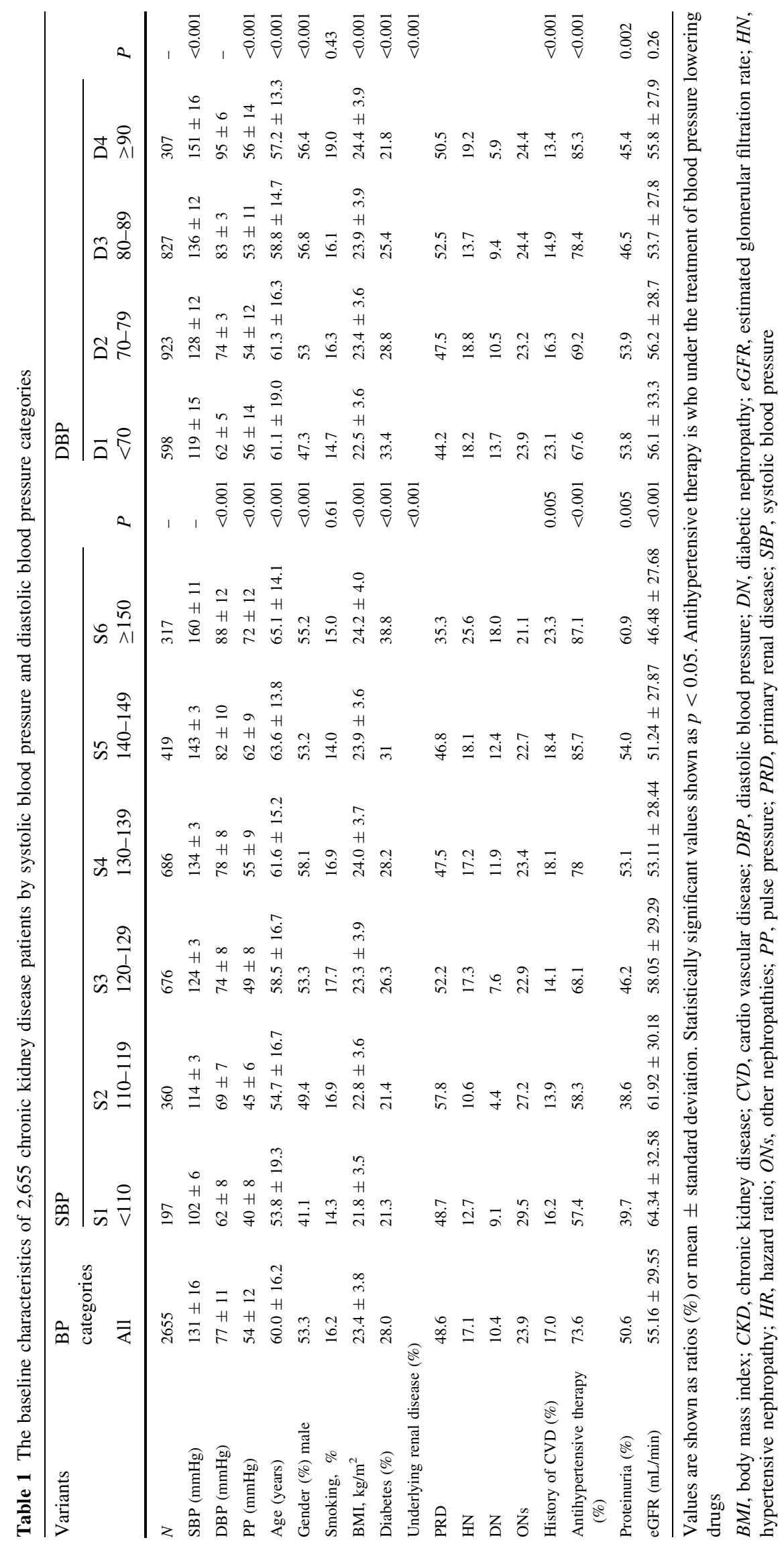




\section{Nonrenal outcome}

During the median follow-up period of 3.02 years [interquartile (IQ) range 1.77-3.12 years], 64 patients died, 120 experienced CVEs, and 225 were not followed up because they had started receiving renal replacement therapy. The incidence rate of the primary end points in each of the BP categories is shown in Fig. 2. The incidence rate of combined CVEs, including cardiac disease, stroke, and allcause mortality, was lowest in the patients in the 110- to 119-mmHg SBP group (S2 group; log-rank $\chi^{2}=7.92$, $p=0.16)$ and in the patients in the 80 - to $89-\mathrm{mmHg}$ DBP group (D3 group; $\chi^{2}=20.0, p<0.001$ ) in the KaplanMeier survival analysis (data not shown). In the subanalysis, higher incidence rates in the lower DBP levels were clearly observed in the patients with proteinuria $\left(X^{2}=64.5, p<0.001\right.$; Fig. 3a), the elderly $\left(X^{2}=141\right.$, $p<0.001$; Fig. 3b), those with a history of CVD ( $X^{2}=226, p<0.001$; Fig. 3c), and those with diabetes $\left(X^{2}=128, p<0.001\right.$; Fig. $\left.3 \mathrm{~d}\right)$. The Cox regression analysis showed that the patients with SBPs $<110 \mathrm{mmHg}$ (S1 group) or DBPs $<70 \mathrm{mmHg}$ (D1 group) were associated with an increased risk of combined CVEs compared with the reference levels in the adjustment model for potential confounders, age, sex, presence of diabetes, eGFR $<60 \mathrm{~mL} / \mathrm{min}$, presence of proteinuria, and history of CVD (HR, 95\% CI 1.85, 1.04-3.30 and 1.65, 1.04-2.62, respectively) as shown in Table 2 (Model 2) and Fig. 4. Further adjustment within the causal pathway of hypertension, antihypertensive therapy, and PP did not modify the significant associations of combined CVEs in the S1 or D1 groups (Table 2).

\section{Renal outcomes}

The relationship between BP and risk of ESKD was examined. During the follow-up period, 225 patients started receiving renal replacement therapy. The Kaplan-Meier survival analysis showed that the incidence rate of ESKD increased progressively in the patients with higher SBP levels ( $\chi^{2}=73.7, p<0.001$; data not shown). In the subanalysis, the higher incidence rate in the higher SBP levels was observed in the patients with positive for proteinuria $\left(X^{2}=298, p<0.001\right.$; Fig. 3e), the elderly $\left(X^{2}=84.8, p<0.001\right.$; Fig. 3f), those with a history of CVD $\left(X^{2}=92.1, p<0.001\right.$; Fig. $\left.3 g\right)$, and those with diabetes $\left(X^{2}=129, p<0.001 ;\right.$ Fig. $\left.3 \mathrm{~h}\right)$. The Cox regression analysis showed that the 140- to 149-mmHg (S5 group) and $\geq 150-\mathrm{mmHg}$ SBP groups (S6 group) were significantly associated with an increased risk of ESKD compared with the reference level in the adjustment model for potential confounders [HR, $1.57(1.07-2.31)$ and 2.04
Fig. 2 The incidence rate of each primary end point according to blood pressure category. The incidence rates (per 1,000 persons per year) of end-stage kidney disease (ESKD) (a), all-cause mortality (b), cardiac disease (c), and stroke (d)
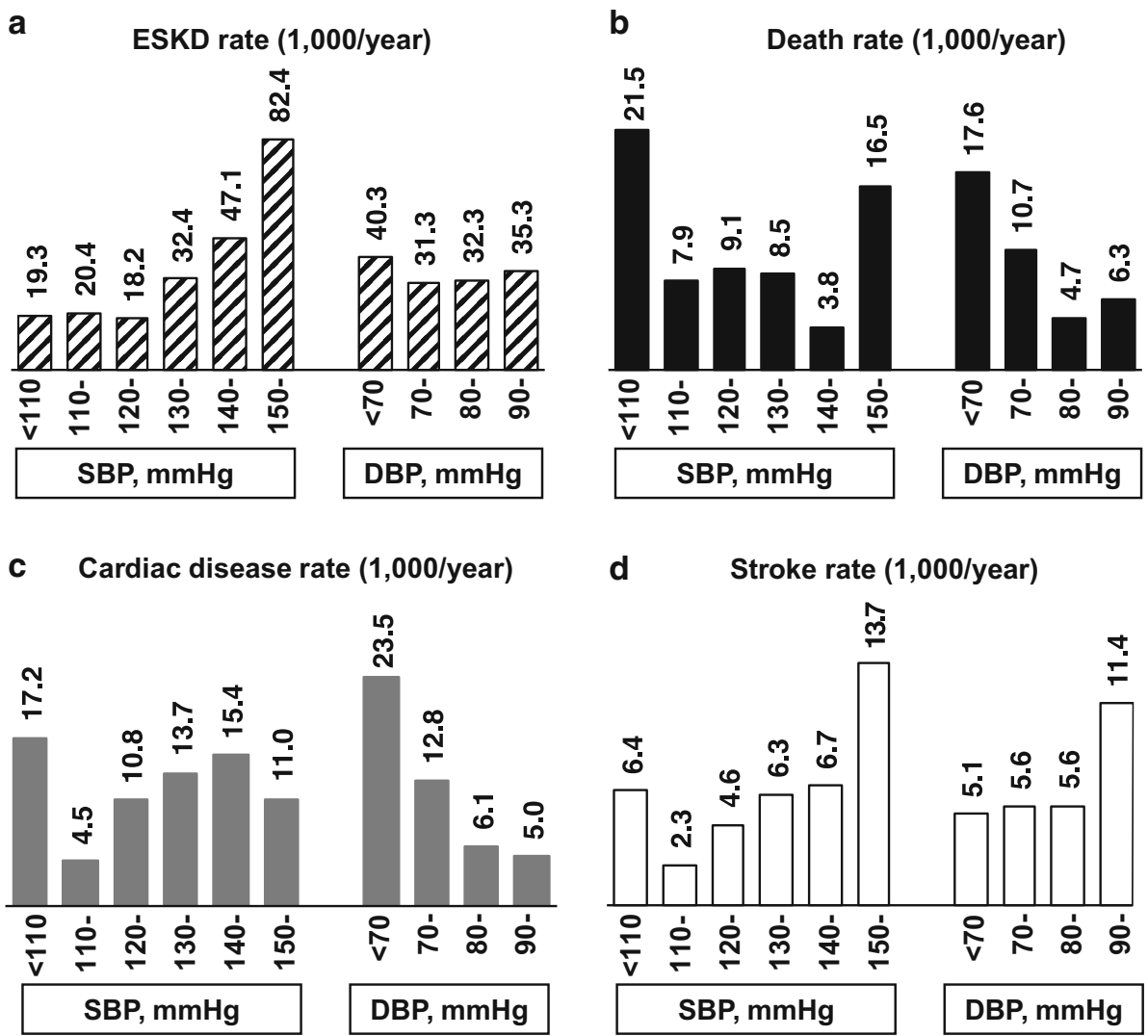

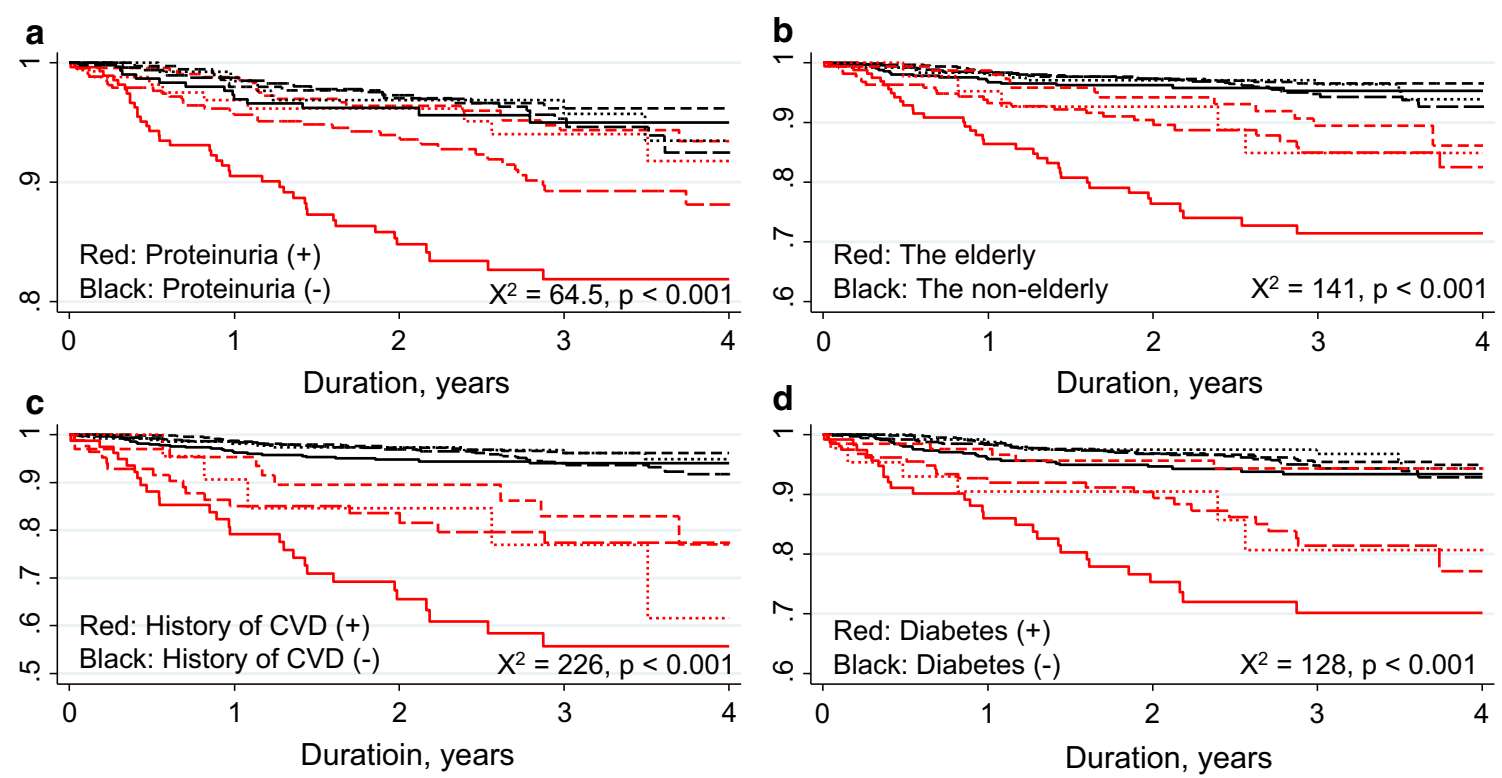

\begin{tabular}{|c|c|c|c|}
\hline \multicolumn{4}{|c|}{ DBP categories } \\
\hline & D1 $(<70 \mathrm{mmHg})$ & --- & D2 $(70-79 \mathrm{mmHg})$ \\
\hline----- & D3 $(80-89 \mathrm{mmHg})$ & (n........... & D4 $(\geq 90 \mathrm{mmHg})$ \\
\hline
\end{tabular}
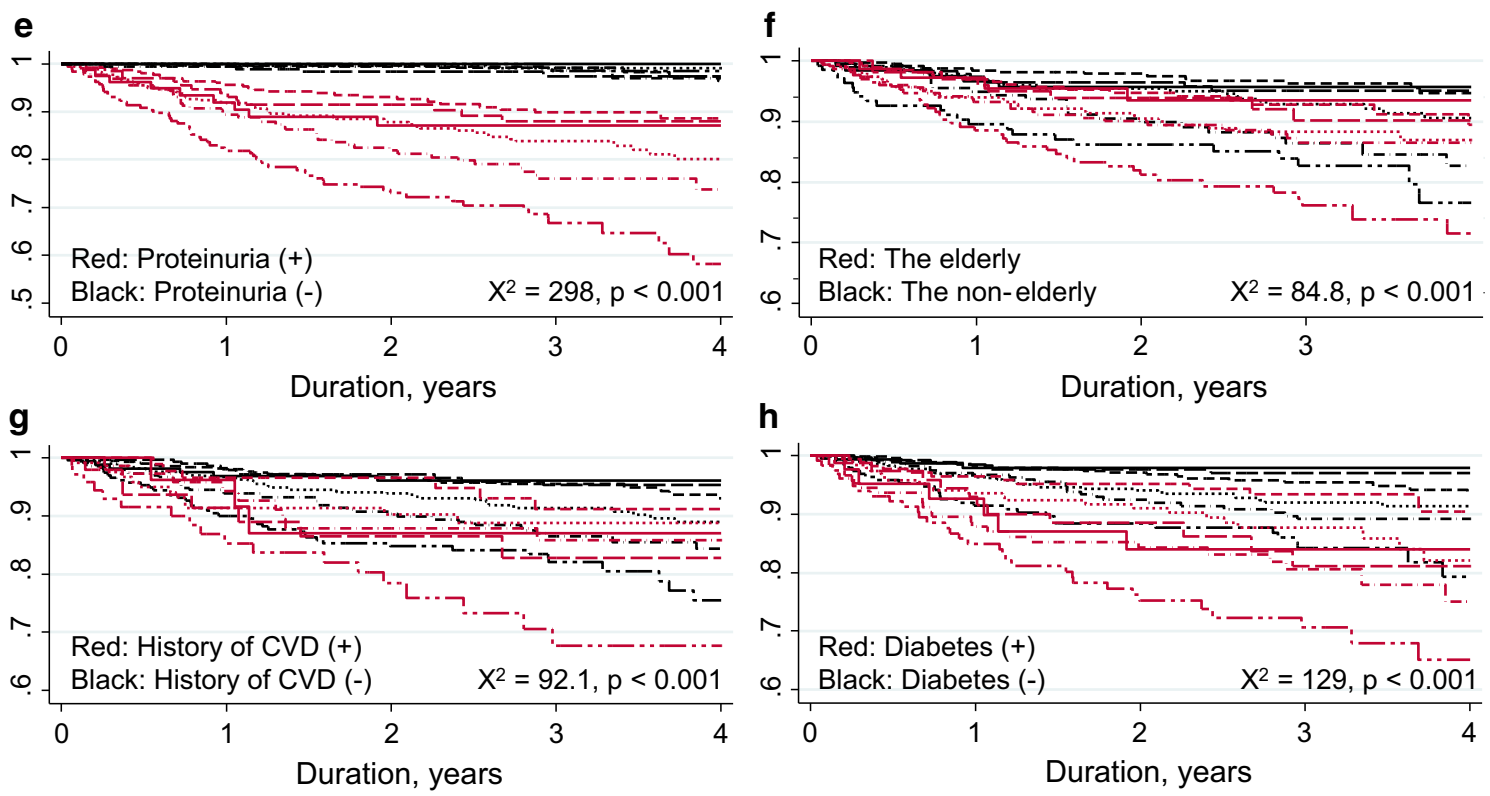

\begin{tabular}{|c|c|c|c|}
\hline \multicolumn{4}{|c|}{ SBP categories } \\
\hline & $\mathbf{S 1}(<110 \mathrm{mmHg})$ & -- & S2 $(110-119 \mathrm{mmHg})$ \\
\hline----- & S3 $(120-129 \mathrm{mmHg})$ & & S4 $(130-139 \mathrm{mmHg})$ \\
\hline$---\cdot-$ & S5 $(140-149 \mathrm{mmHg})$ & $-\cdots$ & $\mathbf{S 6}(\geq 150 \mathrm{mmHg})$ \\
\hline
\end{tabular}

Fig. 3 The sub analysis of event-free survival for nonrenal (a-d) and renal outcomes $(\mathbf{e}-\mathbf{h})$ between the higher-risk subjects (red) and the other subjects (black). Higher incidence rates of nonrenal outcomes in lower diastolic blood pressure (DBP) and renal outcomes in higher

(1.41-2.95), respectively] as shown in Table 3 (Model 2). Meanwhile, SBP levels $<140 \mathrm{mmHg}$, including $\mathrm{SBP}<110 \mathrm{mmHg}$, were associated with lower rates of systolic blood pressure (SBP) were clearly observed in the higher-risk subjects (i.e., patients with proteinuria) $(\mathbf{a}, \mathbf{e})$, the elderly $(\mathbf{b}, \mathbf{f})$, and those with a history of cardiovascular disease $(\mathbf{c}, \mathbf{g})$ and diabetes $(\mathbf{d}$, h)

renal events. Although further adjustment for the antihypertensive therapy did not affect these findings, the increased risk of ESKD in the S5 and S6 groups was not 
Table 2 The Cox proportional hazards models for nonrenal outcomes among the systolic blood pressure or diastolic blood pressure groups in 2,655 patients with chronic kidney disease

\begin{tabular}{|c|c|c|c|c|c|c|c|c|c|}
\hline & BP categories & Model 1 & $95 \% \mathrm{CI}$ & Model 2 & $95 \%$ CI & Model $2+\mathrm{T}$ & $95 \% \mathrm{CI}$ & Model $2+$ PP & $95 \% \mathrm{CI}$ \\
\hline \multicolumn{10}{|c|}{$\mathrm{SBP}(\mathrm{mmHg})$} \\
\hline S1 & $<110$ & 1.99 & $1.12-3.55$ & 1.85 & $1.04-3.30$ & 1.84 & $1.03-3.28$ & 2.14 & $1.13-4.08$ \\
\hline $\mathrm{S} 2$ & $110-119$ & 0.87 & $0.46-1.62$ & 0.84 & $0.44-1.60$ & 0.82 & $0.43-1.56$ & 0.93 & $0.47-1.83$ \\
\hline S3 & $120-129$ & 1.02 & $0.65-1.58$ & 0.99 & $0.63-1.55$ & 0.98 & $0.62-1.53$ & 1.06 & $0.67-1.70$ \\
\hline S4 & $130-139$ & 1 & - & 1 & - & 1 & - & 1 & - \\
\hline S5 & $140-149$ & 0.92 & $0.56-1.52$ & 0.90 & $0.54-1.49$ & 0.92 & $0.55-1.52$ & 0.83 & $0.49-1.41$ \\
\hline S6 & $\geq 150$ & 1.09 & $0.66-1.81$ & 0.93 & $0.56-1.55$ & 0.94 & $0.56-1.57$ & 0.79 & $0.43-1.44$ \\
\hline \multicolumn{10}{|c|}{$\mathrm{DBP}(\mathrm{mmHg})$} \\
\hline D1 & $<70$ & 1.93 & $1.23-3.02$ & 1.65 & $1.04-2.62$ & 1.62 & $1.02-2.58$ & 1.66 & $1.04-2.64$ \\
\hline D2 & $70-79$ & 1.28 & $0.83-1.98$ & 1.30 & $0.83-2.03$ & 1.28 & $0.82-2.00$ & 1.30 & $0.83-2.04$ \\
\hline D3 & $80-89$ & 1 & - & 1 & - & 1 & - & 1 & - \\
\hline D4 & $\geq 90$ & 1.46 & $0.79-2.71$ & 1.51 & $0.81-2.80$ & 1.53 & $0.82-2.85$ & 1.52 & $0.82-2.83$ \\
\hline
\end{tabular}

S4 or D3 served as reference. Cox proportional hazards analysis was performed, and statistically significant values shown as $p<0.05$. Model 1 was adjusted for age and sex, Model 2 was adjusted for age, sex, diabetes, eGFR $<60 \mathrm{~mL} / \mathrm{min}$, proteinuria and a history of CVD. Further adjustments for Model 2 plus antihypertensive therapy (Model $2+\mathrm{T}$ ), and for Model 2 plus pulse pressure (Model $2+\mathrm{PP}$ ) were performed $95 \% C I, 95 \%$ confidence interval; $B P$, blood pressure; $C K D$, chronic kidney disease; $C V D$, cardio vascular disease; $D B P$, diastolic blood pressure; $e G F R$, estimated glomerular filtration rate; $H R$, hazard ratio; $P P$, pulse pressure; $S B P$, systolic blood pressure; $T$, antihypertensive therapy

a

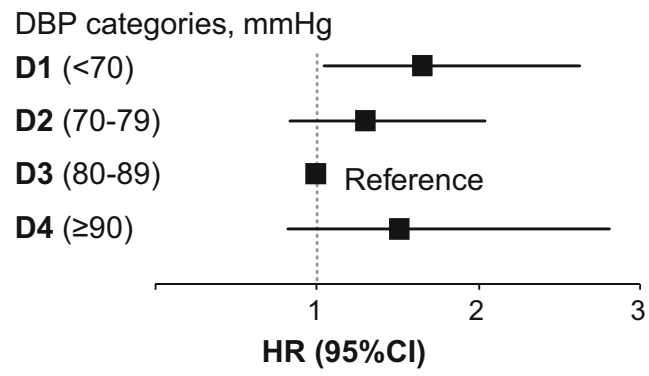

b $\mathrm{SBP}$ categories, $\mathrm{mmHg}$

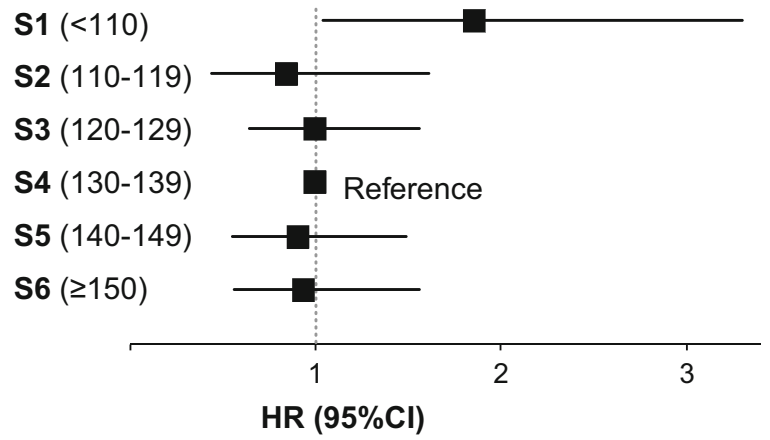

Fig. 4 The relative risks of nonrenal outcomes between systolic blood pressure (SBP) (a) and diastolic blood pressure (DBP) categories (b) are presented in the adjustment models for age, sex, diabetes, estimated glomerular filtration rate, presence of proteinuria, and a history of cardiovascular disease. The patients with SBPs $<110$ and DBPs $<70 \mathrm{mmHg}$ were associated with a significant high risk

observed after adjustment for PP (Table 3). DBP levels had no clear impact on ESKD incidence in the model adjusted only for age and sex (Model 1, Table 3).

\section{Discussion}

Aggressive BP control has been reported to effectively prevent the progressive decline in kidney function [9]; however, the lower limit of the optimal BP target for the prevention of CVD in Asian patients with CKD remains unclear. Therefore, the present study aimed to address 2 aspects of the issue regarding BP in Japanese patients with $\mathrm{CKD}$, namely the risk of renal and nonrenal events. Of note, all the participants in the present study were under nephrologist care at hospitals that offer nephrology services in an outpatient basis, including standard therapeutic interventions (e.g., treatment with hypertensive agents such as renin-angiotensin system inhibitor and/or calciumchannel blockers as needed). Thus, BP was rather well controlled (mean BP 131.0 $\pm 16.2 / 76.7 \pm 10.9 \mathrm{mmHg}$ ), and the rate of outcomes was low, which may reflect a general pattern for Japanese CKD under nephrologist care. In this observational study, lower BP $(\mathrm{SBP}<110 \mathrm{mmHg}$ and DBP $<70 \mathrm{mmHg}$ ) was shown to be associated with an increased risk of nonrenal events, whereas no lower BPs were found to be significant risks of progression to ESKD. It is interesting that these associations of BP in our population were similar with that in American patients with CKD under nephrologist care [18].

We showed that $\mathrm{SBP}<110$ and $\mathrm{DBP}<70 \mathrm{mmHg}$ were independent risk factors of nonrenal events in the patients with well-controlled CKD. Because this finding persisted even after adjustment for other established risk factors of CVD, low BPs were expected to be related to 
Table 3 The Cox proportional hazards models for end-stage kidney disease among the systolic blood pressure or diastolic blood pressure groups in 2,655 patients with chronic kidney disease

\begin{tabular}{|c|c|c|c|c|c|c|c|c|c|}
\hline & BP categories & Model 1 & $95 \% \mathrm{CI}$ & Model 2 & $95 \% \mathrm{CI}$ & Model $2+\mathrm{T}$ & $95 \%$ CI & Model $2+$ PP & $95 \%$ CI \\
\hline \multicolumn{10}{|c|}{$\mathrm{SBP}(\mathrm{mmHg})$} \\
\hline $\mathrm{S} 1$ & $<110$ & 0.69 & $0.34-1.41$ & 0.86 & $0.42-1.75$ & 0.87 & $0.43-1.77$ & 0.86 & $0.78-3.53$ \\
\hline $\mathrm{S} 2$ & $110-119$ & 0.69 & $0.40-1.18$ & 0.94 & $0.55-1.60$ & 0.97 & $0.57-1.66$ & 0.94 & $0.80-2.45$ \\
\hline S3 & $120-129$ & 0.59 & $0.38-0.91$ & 0.67 & $0.43-1.04$ & 0.71 & $0.45-1.09$ & 0.67 & $0.56-1.37$ \\
\hline S4 & $130-139$ & 1 & - & 1 & - & 1 & - & 1 & - \\
\hline S5 & $140-149$ & 1.44 & $0.98-2.12$ & 1.57 & $1.07-2.31$ & 1.54 & $1.05-2.27$ & 0.89 & $0.83-1.84$ \\
\hline S6 & $\geq 150$ & 2.44 & $1.69-3.52$ & 2.04 & $1.41-2.95$ & 2.00 & $1.38-2.89$ & 0.80 & $0.70-1.73$ \\
\hline \multicolumn{10}{|c|}{ DBP (mmHg) } \\
\hline D1 & $<70$ & 1.21 & $0.85-1.74$ & 1.44 & $1.00-2.08$ & 1.48 & $1.02-2.14$ & 1.21 & $0.83-1.75$ \\
\hline D2 & 70-79 & 0.92 & $0.66-1.28$ & 1.15 & $0.82-1.61$ & 1.18 & $0.84-1.65$ & 1.05 & $0.75-1.48$ \\
\hline D3 & $80-89$ & 1 & - & 1 & - & 1 & - & 1 & - \\
\hline D4 & $\geq 90$ & 1.12 & $0.72-1.74$ & 1.28 & $0.83-2.00$ & 1.26 & $0.81-1.96$ & 1.04 & $0.67-1.63$ \\
\hline
\end{tabular}

S4 or D3 served as reference. Cox proportional hazards analysis was performed, and statistically significant values shown as $p<0.05$. Model 1 was adjusted for age and sex. Model 2 was adjusted for age, sex, diabetes, eGFR $<60 \mathrm{~mL} / \mathrm{min}$, proteinuria and a history of CVD. Further adjustments for Mode 2 plus antihypertensive therapy (Model $2+\mathrm{T}$ ), and for Model 2 plus pulse pressure (Model $2+\mathrm{PP}$ ) were performed $95 \% C I, 95 \%$ confidence interval; $B P$, blood pressure; $C K D$, chronic kidney disease; $C V D$, cardio vascular disease; $D B P$, diastolic blood pressure; $e G F R$, estimated glomerular filtration rate; $H R$, hazard ratio; $P P$, pulse pressure; $S B P$, systolic blood pressure; $T$, antihypertensive therapy

combined CVE development. The low BP or aggressive BP-lowering treatment, which may contribute to a reduction in coronary blood flow regulated by DBP and to a failed cardiac function accompanying the low output [19], resulted in an increased risk of cardiac events. Moreover, the possibility of increased ischemic cerebral infarction has been reported in patients with severe atherosclerosis and low BP, such as the elderly and those with diabetes [20]. In the present study, stroke occurred in 41 patients (34.2\%); cardiac disease, in 79 (65.8\%); and cerebral hemorrhage, in $13(10.8 \%)$ of the patients with stroke. The incidence of stroke, especially cerebral hemorrhage, which is closely associated with BP, was lower than that of cardiac disease among the patients with CVEs. Therefore, the incidence of stroke was high in the patients with SBPs $<150 \mathrm{mmHg}$ (Fig. 2d), but the risk of nonrenal events might have not been increased significantly in the Cox models (Table 2). Of note, in the subanalysis, a higher mortality rate was observed in the patients with lower DBP levels, especially in the high-risk populations for CVD, such as the elderly, those with diabetes, those with a CVD history, and those with proteinuria (Fig. 3a-d), which is supported by the previous reports [7, 21-23]. The effect of the adjustment for antihypertensive therapy was minimal (Tables 2, 3). These results suggest the importance of considering the medical background of each patient, and the risks of cardiac disease and infarctions for the management of BP in Japanese patients with CKD. However, the present study was not adequately statistically powered to determine each outcome, owing to a rather small number of events.

Regarding renal outcomes, previous literature reported that lower BP was related to better renal outcomes [9]. In the present study, $\mathrm{SBP} \geq 140 \mathrm{mmHg}$ was associated with an increased risk of renal outcomes, and we did not clearly observe lower BPs at which renal benefits were lost. Although the risk in the patients with SBPs $<110 \mathrm{mmHg}$ increased in the further adjustment models (Model 2, Model $2+\mathrm{T}$ in Table 3), we expect it is because of PP, which clearly affected the risk also in those with higher SBPs, as shown in Model $2+$ PP. In addition, the literature suggests that SBPs $<110 \mathrm{mmHg}$ were associated with increased renal outcomes in the subgroup with proteinuria $>1$ g/day [24], whereas the results of the present study suggest that a BP target $<130 /$ $80 \mathrm{mmHg}$ did not increase the risk of renal outcomes, even in the patients with proteinuria (Fig. 3e). Our study suggests that the safe BP target to prevent renal and nonrenal outcomes differed in the CKD patients, and the lower target BP needs to be determined based on the nonrenal medical background.

This study has some limitations. First, this study had a prospective observational design, without a clear definition set for BP management. Thus, determining the lower BP limit from the present results is difficult. Second, the BP data were based on casual BP measurements at enrollment in the study; the follow-up and home BP monitoring data, 
and the effects of masked and white-coat hypertension were not considered. Third, certain therapeutic agents such as AST-120 (Kremezin) that might have reduced the progression of kidney disease or renin-angiotensin system inhibitor that might be used to reduce the severity of proteinuria can be used regardless of the presence of hypertension. Fourth, renal outcomes were evaluated at commencement of dialysis, and the exact changes in kidney function were not examined in this study. These limitations should be considered when interpreting our data, and further studies are needed to confirm our findings.

\section{Conclusion}

The present study aimed to investigate the adequate range of lower BPs in Japanese patients with CKD under nephrologist care. Our results suggest that no lower BPs significantly increased the risk of progression to ESKD and that SBPs $<110$ and DBPs $<70 \mathrm{mmHg}$ were independent risk factors of CVEs and all-cause mortality. Thus, as a lower limit of BP resulted in increased nonrenal outcome, the lower recommended target is $\geq 110 / 70 \mathrm{mmHg}$ for the management of Asian outpatients with CKD.

Acknowledgments This study was supported by grants from Astellas Pharm Inc. and the Miyagi Kidney Foundation. The authors express special thanks to Ms. Makiko Nakayama and to Ms. Ayuko Momoi for administrative and secretarial assistance. Study contributors: Yuji Yamaguchi (Japanese Red Cross Sendai Hospital), Katsuya Obara (Tohoku Kosai Hospital), Isao Kurihara (Tohoku Kosai Miyagino Hospital), Yasumichi Kinoshita and Kazuto Sato (Japanese Red Cross Ishinomaki Hospital), Kazuro Kaise (Sendai Medical Center), Akira Sugiura and Masahiro Miyata (Osaki Citizen Hospital), Kazuhisa Takeuchi (Koujinkai Central Clinic), Kenji Nakayama and Naoki Akiu (Sendai City Hospital) and Tetsuya Otaka (Katta General Hospital).

Conflict of interest The authors have declared that no conflict of interest exists.

\section{References}

1. Go AS, Chertow GM, Fan D, et al. Chronic kidney disease and the risks of death, cardiovascular events, and hospitalization. N Engl J Med. 2004;351:1296-305.

2. Ogihara T, Saruta T, Rakugi H, et al. Relationship between the achieved blood pressure and the incidence of cardiovascular events in Japanese hypertensive patients with complications: a sub-analysis of the CASE-J trial. Hypertens Res. 2009;32:248-54.

3. Kidney Disease: Improving Global Outcomes (KDIGO) Blood Pressure Work Group. KDIGO Clinical Practice Guideline for the Management of Blood Pressure in Chronic Kidney Disease. Kidney Int. 2012;2(Suppl 8).

4. Peterson JC, Adler S, Burkart JM, et al. Blood pressure control, proteinuria, and the progression of renal disease. The Modification of Diet in Renal Disease Study. Ann Intern Med. 1995;123:754-62.
5. Appel LJ, Wright JT Jr, Greene T, et al. Intensive blood-pressure control in hypertensive chronic kidney disease. N Engl J Med. 2010;363:918-29.

6. Banach M, Aronow WS. Blood pressure j-curve: current concepts. Curr Hypertens Rep. 2012;14:556-66.

7. Sleight $P$, Redon J, Verdecchia $P$, et al. Prognostic value of blood pressure in patients with high vascular risk in the Ongoing Telmisartan Alone and in combination with Ramipril Global Endpoint Trial study. J Hypertens. 2009;27:1360-9.

8. Weiner DE, Tighiouart H, Levey AS, et al. Lowest systolic blood pressure is associated with stroke in stages 3 to 4 chronic kidney disease. J Am Soc Nephrol. 2007;18:960-6.

9. Lv J, Ehteshami P, Sarnak MJ, et al. Effects of intensive blood pressure lowering on the progression of chronic kidney disease: a systematic review and meta-analysis. CMAJ. 2013;185:949-57.

10. Kim AS, Johnston SC. Global variation in the relative burden of stroke and ischemic heart disease. Circulation. 2011;124:314-23.

11. Hyun KK, Huxley RR, Arima H, et al. A comparative analysis of risk factors and stroke risk for Asian and non-Asian men: the Asia Pacific cohort studies collaboration. Int J Stroke. 2013;8:606-11.

12. Lawes CM, Rodgers A, Bennett DA, et al. Blood pressure and cardiovascular disease in the Asia Pacific region. J Hypertens. 2003;21:707-16.

13. Ninomiya T, Perkovic V, Gallagher M, et al. Lower blood pressure and risk of recurrent stroke in patients with chronic kidney disease: PROGRESS trial. Kidney Int. 2008;73:963-70.

14. Nakayama M, Sato T, Sato H, et al. Different clinical outcomes for cardiovascular events and mortality in chronic kidney disease according to underlying renal disease: the Gonryo study. Clin Exp Nephrol. 2010;14:333-9.

15. Nakayama M, Sato T, Miyazaki M, et al. Increased risk of cardiovascular events and mortality among non-diabetic chronic kidney disease patients with hypertensive nephropathy: the Gonryo study. Hypertens Res. 2011;34:1106-10.

16. Special report from the National Institute of Neurological. Disorders and Stroke. Classification of cerebrovascular diseases III. Stroke. 1990;21:637-76.

17. Matsuo S, Imai E, Horio M, et al. Revised equations for estimated GFR from serum creatinine in Japan. Am J Kidney Dis. 2009;53:982-92.

18. Agarwal R. Blood pressure components and the risk for end-stage renal disease and death in chronic kidney disease. Clin J Am Soc Nephrol. 2009;4:830-7.

19. Reynolds JC, Salcido DD, Menegazzi JJ. Coronary perfusion pressure and return of spontaneous circulation after prolonged cardiac arrest. Prehosp Emerg Care. 2010;14:78-84.

20. Mann JF, Anderson C, Gao P, et al. Dual inhibition of the reninangiotensin system in high-risk diabetes and risk for stroke and other outcomes: results of the ONTARGET trial. J Hypertens. 2013;31:414-21.

21. Cushman WC, Evans GW, Byington RP, et al. Effects of intensive blood-pressure control in type 2 diabetes mellitus. $\mathrm{N}$ Engl $\mathrm{J}$ Med. 2010;362:1575-85.

22. Berl T, Hunsicker LG, Lewis JB, et al. Impact of achieved blood pressure on cardiovascular outcomes in the Irbesartan Diabetic Nephropathy Trial. J Am Soc Nephrol. 2005;16:2170-9.

23. Ogihara T, Saruta T, Rakugi H, et al. Target blood pressure for treatment of isolated systolic hypertension in the elderly: valsartan in elderly isolated systolic hypertension study. Hypertension. 2010;56:196-202.

24. Jafar TH, Stark PC, Schmid CH, et al. Progression of chronic kidney disease: the role of blood pressure control, proteinuria, and angiotensin-converting enzyme inhibition: a patient-level meta-analysis. Ann Intern Med. 2003;139:244-52. 